Z Rheumatol 2011 · 70:99-100

DOI 10.1007/s00393-010-0711-4

(c) Springer-Verlag 2011

\author{
C. Fiehn ${ }^{1} \cdot$ U. Müller-Ladner ${ }^{2}$ \\ ${ }^{1}$ ACURA Rheumazentrum Baden-Baden \\ ${ }^{2}$ Kerckhoff-Klinik GmbH, Abteilung Rheumatologie \\ und Klinische Immunologie, Bad Nauheim
}

\title{
Aktueller Stand bei Methotrexat
}

Methotrexat (MTX) nimmt nun schon seit vielen Jahren eine zentrale Rolle in der Behandlung der rheumatoiden Arthritis (RA) ein. Im rasanten Fortschritt der vergangenen Dekade mit der Entwicklung neuer Substanzen und Wirkansätze ist MTX das Kontinuum im therapeutischen Arsenal des praktisch tätigen Rheumatologen. Die EULAR-Empfehlungen zur Behandlung der RA aus dem Jahre 2010 bestätigen die Praxis: MTX steht als DMARD („disease modifying antirheumatic drug“) der ersten Wahl am Anfang der Therapiekaskade zur Behandlung der RA. Darüber hinaus ist es ein wesentlicher Kombinationspartner der Therapie mit Biologika, da es ihre Wirkung verstärkt.

MTX ist ein Folsäureinhibitor und greift damit in Schlüsselmechanismen des Zellstoffwechsels ein. Die niedrig dosierte Therapie mit MTX hat jedoch keinen zytostatischen, sondern einen immunmodulierenden und entzündungshemmenden Effekt. Wie stark dieser ausgeprägt ist und wie weit doch Zelluntergang zu unerwünschten Wirkungen der Therapie führt, unterliegt einer starken interindividuellen Variabilität, welche durch die genetische Ausstattung des Patienten bestimmt wird. Dies ist auch Gegenstand des Beitrags von Mühl u. Pfeilschifter zu diesem Themenheft, welches Pharmakogenetik und Pharmakogenomik zum Gegenstand hat. In der Arbeit wird deutlich, dass eine ganze Reihe von folsäureabhängigen Enzymen Zielstrukturen von MTX sind. Durch genetische Polymorphismen können diese Enzyme, aber auch Moleküle des MTX-Transports und -Metabolismus die MTX-Wirkungen und Nebenwirkungen beeinflussen.
Noch ist man aber auf der Suche nach soliden Prädiktoren mit praktischer, klinischer Relevanz.

Was die unerwünschten Wirkungen von MTX sein können und welche Rolle sie wirklich spielen, ist das Thema des Beitrags von G. Keyßer. MTX-Toxizität ist nicht nur bedingt durch die Dosis und eine mögliche Akkumulation des Medikaments bei einer verminderten renalen Ausscheidung, auch die individuellen genetischen Voraussetzungen des Patienten, Begleittherapien und Komorbiditäten sind entscheidend für das Risiko, unerwünschte Wirkungen durch MTX zu erleben. Mehr Wissen darüber hilft dabei, diese zu vermeiden und den therapeutischen Nutzen bei möglichst vielen Patienten auszunutzen.

K. Krüger fasst die Daten, welche zur Kombinationstherapie von MTX mit anderen DMARDs oder Biologika existieren, in seinem Artikel zusammen. MTX hat hier einen wesentlichen Stellenwert, und nicht nur die Wirksamkeit, sondern auch die Sicherheit von Kombinationstherapien unter Einschluss von MTX ist gut untersucht. Ob auch Biologika der zweiten Generation weiter in Kombination gegeben werden müssen - bei Tocilizumab wie auch dem JAK-Inhibitor Tasocitinib scheinen Kombinationen mit MTX keine Vorteile zu bringen -, wird in Zukunft weiter untersucht werden.

MTX wird nicht nur bei RA sondern auch bei einer Vielzahl von anderen rheumatischen Erkrankungen eingesetzt. Dabei stützen sich die Indikationen auf mehr oder wenig gute Evidenz, oft aus alten, kleinen und methodisch nicht ausgefeilten klinischen Studien. Trotzdem dürf- te MTX das am häufigsten „off-label“ gegebene Medikament in der Rheumatologie sein. Diese Indikationen von MTX sind das Thema des Artikels von Briem u. Lorenz, der alle Daten und Studien zusammenfasst. Die Autoren können zeigen, dass noch viel mehr klinische Studien notwendig sind, um in der Therapie mit MTX außerhalb der Indikation RA die Spreu vom Weizen zu trennen.

Trotz aller Erfolge der Folatinhibition als Standard der RA-Therapie ist die Substanz MTX seit über 6o Jahren immer noch unverändert. Ob sich das ändern wird und in welcher Form die Therapie mit MTX und anderen Folatinhibitoren Zukunft hat, ist das Thema des letzten Beitrags durch den Gastherausgeber dieses Schwerpunktheftes. Neue Erkenntnisse über den Metabolismus und den Transport von MTX erschließen nun auch Ansätze, dieses Medikament und den Wirkmechanismus weiter zu verbessern. Da$\mathrm{zu}$ gehören die gezielte Beeinflussung des Aktivierungsschrittes der Polyglutamisierung von MTX, die Anreicherung des Wirkstoffs in der Entzündung durch „targeted drug delivery“, z. B. Albumin-gekoppeltes MTX, sowie die Weiterentwicklung von Folatinhibitoren, welche spezifisch den $\beta$-Rezeptor des Folattransports nützen und damit gezielt auf synoviale Makrophagen wirken.

Die Erfolgsgeschichte von MTX und der Folatinhibition zur Behandlung rheumatischer Erkrankungen dauert daher an. Neues Wissen erschließt weitere Möglichkeiten, mit denen dieses erfolgreiche antirheumatische Wirkprinzip weiter entwickelt werden kann. Die Evidenz für dessen Einsatz, die Stärken und Schwächen der 
Substanz und die Bedeutung in der praktischen Rheumatologie sind die Themen des vorliegenden Schwerpunktheftes der Zeitschrift für Rheumatologie.

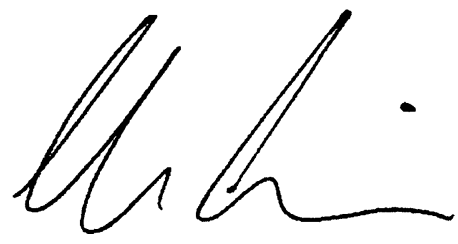

Prof. Dr. C. Fiehn

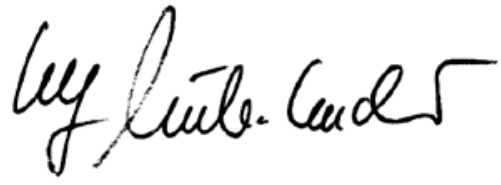

Prof. Dr. U. Müller-Ladner

\section{Korrespondenzadresse}

\section{Prof. Dr. C. Fiehn}

ACURA Rheumazentrum Baden-Baden Rotenbachtalstr. 5, 76530 Baden-Baden c.fiehn@rheumazentrum-baden.de

\section{Joseph Kuhn, Jan Böcken (Hrsg.) Verwaltete Gesundheit} Konzepte der Gesundheitsberichterstattung in der Diskussion.

Frankfurt am Main: Mabuse - Verlag 2009, 319 S., (ISBN 3-940529-46-6), 29.00 EUR

Nach dem Erscheinen von Büchern zur Konzeption des Aufbaus einer Gesundheitsberichterstattung (GBE) zu Beginn und Mitte der 90er Jahre schien es jahrelang so, als ob die GBE damit konsolidiert oder aber auch stagnierend sei. Dieses Buch setzt nun fort, was nach einer allgemeinen,Publikationspause' mit dem 2006 im gleichen Verlag erschienenen Band "Gesundheit zwischen Statistik und Politik" begonnen bzw. wieder aufgenommen wurde: eine Diskussion über Konzeptionen und konzeptionelle Weiterentwicklungen der GBE. Der Band geht auf einen im Sommer 2008 im Kloster Benedictbeuern abgehaltenen Workshop zurück. Historisch wie aktuell, wie in mehreren Beiträgen gezeigt wird, sind das aufklärerische Motiv einer GBE und die Mittel der Demokratisierung und Bürgerorientierung nicht die allein existierenden und bestimmenden Motive auf den Ebenen staatlicher Gesundheitsverwaltung. Die Herausgeber nennen den eigentlichen Kern ein „komplexes Anknüpfen an und Hervorbringen von gesundheitlichen Diskursen", welche insbesondere auch nach machttheoretischen (Eva Barlösius) und ethischen (Joseph Kuhn) Aspekten zu hinterleuchten seien.

Der Eintritt neuer staatlicher, halbstaatlicher und nichtstaatlicher Akteure in die Berichterstattungs- und ReportArena, die weitere Ausdifferenzierung von Berichterstattungsroutinen und die verschiedenen Blickwinkel, die von Institutionen in die GBE eingebracht werden, eröffnen auch neue Schnittstellen zur GBE. Beispielhaft seien hier nur die Beiträge zum Gemeinsamen Bundesausschuss (Matthias Perleth), zur Qualitätsberichterstattung (Enno Swart), zu Gesundheitszielen (Gabriele Klärs und Thomas Ziese), zu Präventionszielen der GKV (Wolfgang Bödeker), zum Gesundheitsmonitor der Bertelsmann-Stiftung (Jan Böcken) oder zur Integration von Bildungs- Sozial- und
Gesundheitsberichterstattung (Waldemar Süß) genannt.

Bei alledem wird der - insbesondere vom Sachverständigenrat im Jahr 1987 eingebrachte - Anspruch aufrechterhalten, GBE solle eine Datengrundlage für sachlich rationale Entscheidungen liefern und die Beteiligung breiterer Kreise der Öffentlichkeit an den gesundheitspolitischen Entscheidungen ermöglichen. So resümiert - bei aller Kenntnis oftmals gegenteilig zustande gekommener gesundheitspolitischer Entscheidungen - Josef Kuhn in seinem Schlusskapitel die Funktionsbestimmung der GBE als eines nicht etwa epidemiologischen, sondern politischen Konzepts (gesundheitspolitisches Modernisierungskonzept, good governance, ,'Stärkung von gesundheitspolitischen gegenüber finanzpolitischen Imperativen"). Eine hierzu auch wohl sinnvolle (wiewohl fraglich erwünschte) Vereinheitlichung des Begriffs der GBE sei wohl nur auf dem rechtlichem Wege einer systematischen Funktionsbestimmung möglich. Allemal jedoch fehlten noch weiter politikwissenschaftliche Analysen zu policy, politics und polity der GBE. Bleibt zu hoffen, dass der Band Beachtung finde und die Fragen weiter auf konzeptioneller Ebene diskutiert und vorangebracht werden.

Prof. Dr. Thomas Elkeles, Hochschule Neubrandenburg, Fachbereich Gesundheit, Pflege, Management 